\title{
Elevated TFAP4 regulates IncRNA TRERNA1 to promote cell migration and invasion in gastric cancer
}

\author{
HUAZHANG WU ${ }^{1,2}$, XIUFANG LIU ${ }^{1}$, PIHAI GONG ${ }^{1}$, WEI SONG ${ }^{1}$, MENGHAN ZHOU ${ }^{1}$, \\ YIPING $\mathrm{LI}^{3}$, ZHUJIANG ZHAO ${ }^{1}$ and HONG FAN ${ }^{1}$ \\ ${ }^{1}$ Department of Medical Genetics and Developmental Biology, Medical School of Southeast University and \\ Key Laboratory of Developmental Genes and Human Diseases, Ministry of Education, Southeast University, \\ Nanjing, Jiangsu 210009; ${ }^{2}$ School of Life Science, Bengbu Medical College, Bengbu, Anhui 233030; \\ ${ }^{3}$ Department of Pathology, Medical School of Southeast University, Nanjing, Jiangsu 210009, P.R. China
}

Received December 1, 2017; Accepted May 15, 2018

DOI: $10.3892 /$ or.2018.6466

\begin{abstract}
Cancer cell invasion and metastasis are the leading causes of the high mortality rates in patients with malignant tumors. There is accumulating evidence to indicate that dysregulated long non-coding RNAs (lncRNAs) may be involved in the progression of tumor invasion and metastasis. However, the regulatory mechanisms of the aberrant expression of lncRNAs remain largely unknown, although the roles of lncRNAs as drivers of tumor suppressive and oncogenic functions have appeared in prevalent cancer types in recent years. In the present study, we identified that the transcription factor, activating enhancer-binding protein 4 (TFAP4), acts as a key modulator of translation regulatory long non-coding RNA 1(TRERNA1), which has been proven to promote the invasion and metastasis of gastric cancer (GC) cells. We revealed that TRERNA1 was upregulated in gastric carcinogenesis and promoted cell migration and invasion in GC. Using bioinformatics analysis, we observed that there were several potential binding sites of TFAP4 in the promoter region of TRERNA1. The knockdown of TFAP4 significantly reduced the expression level of TRERNA1, whereas the ectopic expression of TFAP4 significantly increased the expression level of TRERNA1 in GC cell lines. Dual luciferase reporter assay combined with chromatin immunoprecipitation (ChIP) revealed that TFAP4 specifically regulated the transcriptional activity of TRERNA1 by binding to the E-box motifs in the TRERNA1 promoter. In addition, there was a positive correlation between the TFAP4
\end{abstract}

Correspondence to: Professor Hong Fan, Department of Medical Genetics and Developmental Biology, Medical School of Southeast University and Key Laboratory of Developmental Genes and Human Diseases, Ministry of Education, Southeast University, 87 Dingjiaqiao Road, Nanjing, Jiangsu 210009, P.R. China E-mail: fanh@seu.edu.cn

Key words: activating enhancer-binding protein 4, translation regulatory long non-coding RNA 1, migration and invasion, gastric cancer and TRERNA1 expression level in clinical GC cases, which also indicated that TFAP4 can directly modulate the expression of TRERNA1. In the present study, we provide a novel potential therapeutic target and strategy for GC.

\section{Introduction}

Gastric cancer (GC) is one of the most common human cancers and the second leading cause of cancer-related mortality worldwide, as well as specifically in China (1-3). Despite the fact that the therapeutic strategies against malignant tumors have markedly improved in recent years, the metastasis of malignant tumor cells is the cause of most cancer-related deaths and remains one of the most enigmatic aspects of cancers, including GC $(2,4)$. Therefore, identifying metastasis-associated gene expression and the molecular regulatory mechanisms would provide a promising molecular target for the treatment of cancer.

Long non-coding RNAs (lncRNAs) are transcripts longer than 200 nucleotides, that lack protein coding capacity (5). Accumulating evidence indicates that lncRNAs are involved in cellular apoptosis, cell proliferation, migration and invasion (6-8) through a variety of mechanisms, including chromosome remodeling, RNA processing, localization, mRNA stability, translation and even as a competing endogenous RNA (9-11). The dysregulation of IncRNAs in tumor formation and progression has been investigated, with a particular focus on the mechanisms of action of lncRNAs. To date, the majority of studies have analyzed the functions of lncRNAs, the underlying mechanisms of dysregulated lncRNAs in tumorigenesis and their potential roles as prognostic markers or therapeutic targets for specific cancers (12). However, little is known about the transcriptional regulatory mechanisms responsible for the aberrant transcription of lncRNAs in cancers. In the present study, we characterized the regulatory mechanisms through which the transcription factor, activating enhancer-binding protein 4 (TFAP4), activates the expression of lncRNA TRERNA1, which plays an important role in invasion and metastasis in GC, by binding to its promoter. Thus, the present study may provide a novel potential therapeutic strategy and target for GC. Our findings may also contribute 
to the improvement of individualized treatment decisions for GC patients.

\section{Materials and methods}

Tissue collection and ethics statement. A total of 48 paired fresh gastric cancer tissues and adjacent non-tumorous gastric tissues were analyzed in the present study (clinicopathological characteristics shown in Table I). The patients had undergone surgical resection between May, 2010 and December, 2014 at the Affiliated Jiangyin Hospital of Medical School (Jiangyin, Jiangsu, China) of Southeast University, China. The study was approved by the Research Ethics Committee of Southeast University, and written informed consent was obtained from all patients.

Cell culture. The GC cell lines, AGS, SGC-7901 and BGC-823, were purchased from the Institute of Biochemistry and Cell Biology of the Chinese Academy of Sciences (Shanghai, China). The MKN-74 cell line was purchased from Cellcook Biotech Co., Ltd (Guangzhou, China). Cells were cultured in RPMI-1640 medium supplemented with $10 \%$ fetal bovine serum (FBS; Wisent Inc., St. Bruno, QC, Canada), 100 U/ml penicillin and $100 \mathrm{mg} / \mathrm{ml}$ streptomycin (Invitrogen, Carlsbad, CA, USA) in an incubator humidified air at $37^{\circ} \mathrm{C}$ with $5 \% \mathrm{CO}_{2}$.

Reverse transcription-quantitative PCR (RT-qPCR) assay. Total RNA was first extracted from the cultured cells or tissues using TRIzol reagent (Invitrogen). Subsequently, RNA was reverse transcribed into cDNA using the PrimeScript ${ }^{\mathrm{TM}}$ RT reagent kit with gDNA Eraser (Takara, Dalian, China). Quantitative (real-time) PCR expression analyses were performed using the SYBR ${ }^{\circledR}$ Premix Ex Taq ${ }^{\mathrm{TM}}$ II kit (Takara). The results were normalized to the expression level of $\beta$-actin and all RT-qPCR data were evaluated using the $2^{-\Delta \Delta C q}$ method (13), and their gene-specific primers as follows. TFAP4 forward, 5'-GCAGGCAATCCAGCACAT-3' and reverse, 5'-GGAGGCGGTGTCAGAGGT-3'; $\beta$-actin forward, 5'-AAAGACCTGTACGCCAACAC-3' and reverse, 5'-GTC ATACTCCTGCTTGCTGAT-3'. The RT-qPCR thermocycling conditions for TFAP4 and $\beta$-actin began with an initial hold for $2 \mathrm{~min}$ at $95^{\circ} \mathrm{C}$, followed by 40 cycles of denaturation at $95^{\circ} \mathrm{C}$, annealing at $60^{\circ} \mathrm{C}$ and extension at $72^{\circ} \mathrm{C}$ all for $30 \mathrm{sec}$. Each experiment was performed in triplicate.

Plasmid construction and cell transfection. The cDNA TRERNA1 was synthesized by Genewiz (Suzhou, China) and then cloned into the pcDNA3.1 plasmid (Invitrogen, Carlsbad, CA, USA). The cDNA of TFAP4 was produced by reverse-transcription PCR (RT-PCR) and then cloned into the HindIII/EcoRI sites of the pcDNA3.1 plasmid. The primer sequences for TFAP4 PCR amplification were as follows: Forward, 5'-CCCAAGCTTATGGAGTATTTCATGGTG CC-3' and reverse, 5'-GGTGGAATTCGGGGGGTAGTC AGGGAA-3'. Short hairpin RNA (shRNA) targeting TFAP4 was synthesized by Genewiz, and ligated into the B $g l \mathrm{II} / \mathrm{HindIII}$ sites of the pSUPER-EGFP vector (OligoEngine, Seattle, WA, USA) after annealing. The sequences were as follows: 5'-GAT CCCCGTGATAGGAGGGCTCTGTAGTTCAAGAGACTAC AGAGCCCTCCTATCACTTTTTGGAAA-3' and 5'- AGC TTTTCCAAAAAGTGATAGGAGGGCTCTGTAGTCTCT
Table I. Association of TFAP4 expression with clinicopathological characteristics of patients with GC.

\begin{tabular}{|c|c|c|c|}
\hline \multirow[b]{2}{*}{ Characteristic } & \multicolumn{2}{|c|}{ Expression of TFAP4 } & \multirow[b]{2}{*}{ P-value } \\
\hline & $\mathrm{T}>\mathrm{N}$ & $\mathrm{T} \leq \mathrm{N}$ & \\
\hline Age (years) & & & 0.868 \\
\hline$<60$ & 8 & 2 & \\
\hline$\geq 60$ & 27 & 11 & \\
\hline Sex & & & 1.000 \\
\hline Female & 10 & 5 & \\
\hline Male & 25 & 8 & \\
\hline Lauren classification & & & 0.033 \\
\hline Intestinal type & 12 & 9 & \\
\hline Diffuse type & 23 & 4 & \\
\hline Histological grade & & & 0.506 \\
\hline High & 12 & 5 & \\
\hline Moderate & 7 & 4 & \\
\hline Poor & 16 & 4 & \\
\hline TNM staging & & & 0.675 \\
\hline Stage I/II & 23 & 7 & \\
\hline Stage III/IV & 12 & 6 & \\
\hline Lymph node metastasis & & & $0.021^{\mathrm{a}}$ \\
\hline No & 11 & 9 & \\
\hline Yes & 24 & 4 & \\
\hline
\end{tabular}

Chi-square test, ${ }^{\mathrm{a}} \mathrm{P}<0.05$. T, tumor tissue; $\mathrm{N}$, non-tumor tissue.

TGAACTACAGAGCCCTCCTATCACGGG-3'. Transfection was performed using Lipofectamine 2000 according to the manufacturer's instructions (Invitrogen). For RT-qPCR assays, at $24 \mathrm{~h}$ post-transfection, the cells were lysed and total cellular RNA was extracted using TRIzol reagent (Invitrogen), and the transfection efficiency was determined by RT-qPCR.

Cell migration and invasion assays. Wound healing, cell migration and invasion assays were performed as previously described (14). Briefly, a scratch wound was generated using a $10 \mu \mathrm{l}$ blunt pipette tip, and cells were cultured in serum-free medium, after being washed with phosphate-buffered saline (PBS) to remove floating cells. Cell migration and invasion assays were performed with a Transwell chamber with $8-\mu \mathrm{m}$ pore size (EMD Millipore, Billerica, MA, USA). Cells were seeded into the upper chamber, and the cells migrating through the pores or invading through the Matrigel were fixed and stained with $0.5 \%$ crystal violet (Beyotime Institute of Biotechnology, Nantong, China) following 24-36 h of incubation.

Luciferase reporter assay. The TRERNA1 promoter fragments containing the predicted TFAP4 binding site were amplified by PCR (primers are listed in Table II), and then subcloned into the downstream of the luciferase reporter gene in the pGL3 Luciferase Reporter Vectors (Promega Corporation, Madison, WI, USA). The wild-type sequence with wild-type E2 site 
Table II. Primer sets designed to construct luciferase reporter vectors containing the predicted E-box sequence in the promoter region of the TRERNA1.

\begin{tabular}{lll}
\hline Loci & Primer & \multicolumn{1}{c}{$5^{\prime} \rightarrow 3^{\prime}$} \\
\hline E1 E3 & Sense & ATATGCTAGCTAAGCACACACCACCTTGCC \\
& Antisense & ATATAAGCTTGTTCCCACCCCACTCACAAA \\
E2 & Sense & ATATGCTAGCCACTGACTTTCCGCTTCCC \\
& Antisense & ATATAAGCTTTTAGCACTGGTTCCCTTCT \\
E3 & Sense & ATATGCTAGCCAGGCGTCAGAAGGGAACCA \\
& Antisense & ATATAAGCTTCAACCAGCCAAGGCGGAGTA \\
\hline
\end{tabular}

Table III. Primer sets designed to amplify the conserved E-box sequence in the promoter region of the TRERNA1.

\begin{tabular}{lll}
\hline Loci & \multicolumn{1}{c}{ Primer } & \multicolumn{1}{c}{$5^{\prime} \rightarrow 3^{\prime}$} \\
\hline E1 & Sense (P1) & ATTATAAGCACACACCACC \\
& Antisense (P1) & ATAGCAAAAGAAAAACACC \\
E2 & Sense (P2) & CACTGACTTTCCGCTTCCC \\
& Antisense (P2) & TTAGCACTGGTTCCCTTCT \\
E3 & Sense (P3) & CAGGCGTCAGAAGGGAACCA \\
& Antisense (P3) & CAACCAGCCAAGGCGGAGTA \\
& &
\end{tabular}

(5'-CAGCTG-3') was 5'-CTAGAGGCACTGACTTTCCGC TTCCCAGCTGTGTGAGGTCTTGCTCAATTTC-3', and the mutant-type sequence with mutant-type E2 site (5'-TGA TCA-3') was 5'-CTAGAGGCACTGACTTTCCGCTTCCTG ATCATGTGAGGTCTTGCTCAATTTC-3'. These sequences were synthesized by Genewiz and subcloned into the pGL3 plasmid, separately. The luciferase activities were determined using a dual luciferase assay kit (Promega Corporation) according to the manufacturer's protocol. The relative luciferase activity was normalized to the Renilla luciferase activity.

Chromatin immunoprecipitation (ChIP) assay. ChIP assays were performed using the ChIP kit (EMD Millipore) according to the manufacturer's instructions in MKN-74 cells. Antibodies against TFAP4 [AP-4(C-18)X, dilution 1:500] and normal mouse IgG were obtained from Santa Cruz Biotechnology (Santa Cruz, CA, USA). Primers designed to amplify the conserved E-box in the promoter of the TRERNA1 are listed in Table III.

Statistical analysis. Statistical analyses were performed using SPSS 20.0 software (IBM Corp., Armonk, NY, USA) or GraphPad Prism 5 software (GraphPad Software, Inc., La Jolla, CA, USA). The significance of differences in the TFAP4-dependent regulation of TRERNA1 expression and transcriptional activity of E-box (E1-E3, E2 and E3) of the TRERNA1 promoter region between TFAP4 and control groups were estimated using a Student's t-test. The association between TFAP4 expression and pathological features of GC was analyzed with the Chi-square $\left(\chi^{2}\right)$ test. The correlation between the TFAP4 and TRERNA1 expression levels was analyzed using Pearson's correlation coefficient test. All $\mathrm{P}$-values presented were two-sided and $\mathrm{P}<0.05$ was considered to indicate a statistically significant difference.

\section{Results}

TFAP4 upregulates the expression level of IncRNA TRERNA1. Transcription factors are the most important regulators of gene transcription by promoting or blocking specific genes (15). In order to clarify the transcription factors that may contribute to the regulation of TRERNA1, in this study, we first screened the potential transcription factors and their possible binding sites by scanning the TRERNA1 gene promoter from upstream $-4 \mathrm{~kb}$ to the downstream $+1 \mathrm{~kb}$ region through the online analytical website (http:/gene-regulation.com/pub/databases. html). The predicted results indicated that a variety of transcription factors, such as TFAP4, CCAAT enhancer binding protein beta (CEBPB), heat shock factor 1 (HSF1), Jun proto-oncogene (JUN), nuclear factor (NFE2L2) and cAMP response element binding protein 1 (CREB1) could bind to the promoter region of TRERNA1 (data not shown). TFAP4 was considered as a priority candidate, since there were multiple binding sites in the vicinity to the transcription start site (TSS) of TRERNA1 (Fig. 1A).

In order to clarify the effect of TFAP4 on the expression of TRERNA1, we constructed TFAP4 overexpression and silencing vectors, and transfected these constructs into GC cells. As displayed in Fig. 1B, the enhanced expression of TFAP4 markedly increased the expression level of TRERNA1 in SGC-7901 and AGS cells. In addition, we observed a significant decrease in the TRERNA1 level following the silencing of TFAP4 in MKN-74 and BGC-823 cells (Fig. 1C). These results indicated that the expression of TRERNA1 was regulated by TFAP4 in GC cell lines.

TFAP4-regulated transcriptional activity of TRERNA1 depends on the E-box of its promoter. To determine whether the TFAP4-dependent regulation of the expression of TRERNA1 is attributed to activated transcription, a series of constructs containing different fragments of E-box (E1-E3, E2 and E3) of the TRERNA1 promoter region were generated (Fig. 2A). As displayed in Fig. 2B, the overexpression of TFAP4 significantly enhanced TRERNA1 gene promoter activity in the SGC-7901 and BGC-823 cells, particularly in 
A

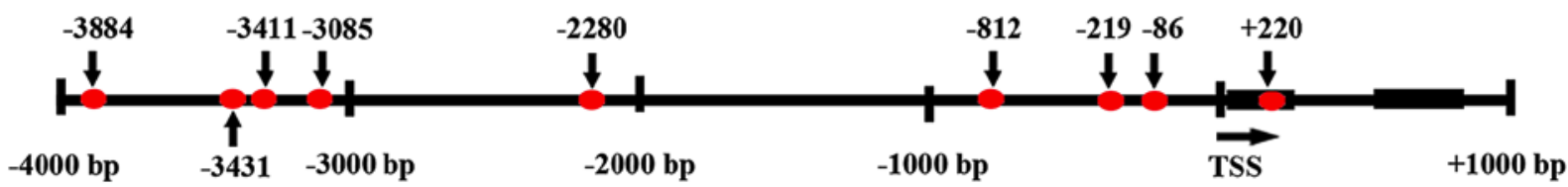

TRERNA1 1000 bp

B

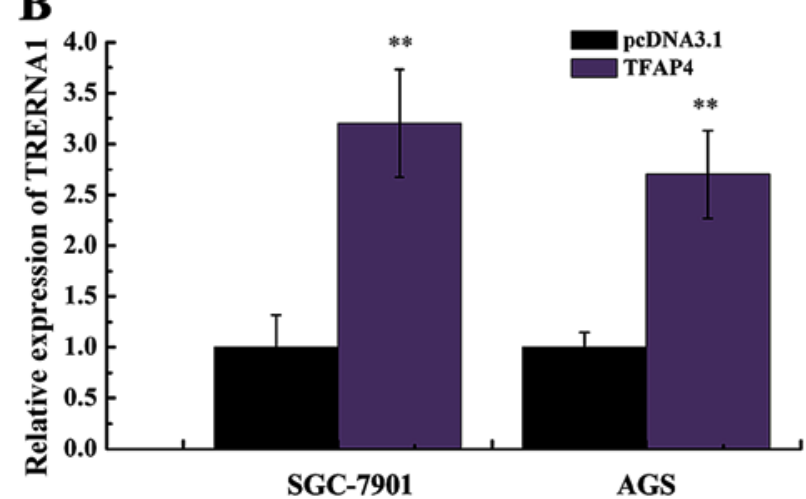

C

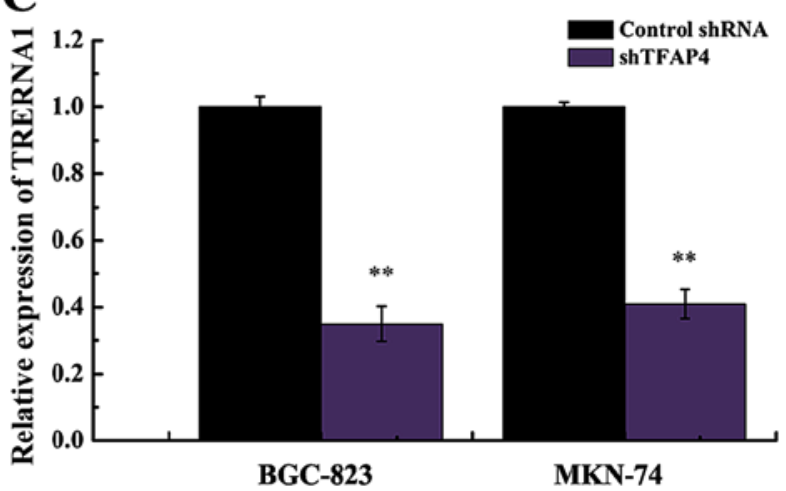

Figure 1. TFAP4 regulates the expression of TRERNA1 in GC cells. (A) The potential binding sites (E-box) of TFAP4 in the promoter region of the TRERNA1 gene. (B) Enforced expression of TFAP4 significantly promoted the transcription of TRERNA1 compared with the control group (pcDNA3.1) in SGC-7901 and AGS cells. (C) Compared with the control shRNA group, the expression level of TRERNA1 was significantly decreased after silencing TFAP4 gene in BGC-823 and MKN-74 cells. Vertical columns and error bars represent mean $\pm \mathrm{SD}$. ${ }^{* *} \mathrm{P}<0.01$. GC, gastric cancer. TFAP4, activating enhancer-binding protein 4; GC, gastric cancer.

the E1-E3 fragment containing three TFAP4 binding sites in the TRERNA1 promoter (Fig. 2B).

A previous study revealed that promoters activated by TFAP4 are located closer to the TSS than those present at TFAP4-suppressed promoters (16). In this study, to further characterize the role of the E-box motif situated on TRERNA1 as a transcriptional activator, we selected the E2 site to construct the dual luciferase reporter vector containing the motif of both the wild- (CAGCTG) and mutant-type (TGATCA) at the E2 sites (Fig. 2C). Dual-luciferase assays revealed that the overexpression or silencing of TFAP4 significantly affected the transcriptional activity of the wild-type E2 sites in SGC-7901, BGC-823 and MKN-74 cells (Fig. 2D and E). In contrast to the wild-type, TFAP4 had no significant impact on the activity of the E2-mutant TRERNA1 promoter in GC cells (Fig. 2D and E). These results also demonstrated that mutations abolished TFAP4 binding to E2. Collectively, our results revealed that TFAP4 regulation of the transcriptional activity of TRERNA1 was dependent on the CACGTG motifs in the TRERNA1 promoter.

TFAP4 specifically binds to the E-box motif of TRERNAI promoter in vitro. Previous studies have revealed that TFAP4 is effective for both the suppression and/or activation of target genes. TFAP4 downregulates the expression level of p16 and p21 to suppress cell senescence by directly binding the putative E-box site (CAGCTG) $(16,17)$. In this study, in order to determine whether TFAP4 promotes TRERNA1 transcriptional activity by directly binding to its promoter, we designed PCR primers (Table III) specific for the amplification of the three E-box sites (E1, E2 and E3) in the TRERNA1 promoter region (Fig. 3A). As displayed in Fig. 3B, the results indicated that TFAP4 bound to the promoters of TRERNA1 in the vicinity of their E-box binding motifs E1, E2 and E3, which reflected specificity of the TFAP4 binding to the E-box motifs located in the promoter of TRERNA1 (Fig. 3B). Notably, ChIP different enrichment observed among E1, E2 and E3 (Fig. 3B), indicated that the maximal binding capacity of TFAP4 to the TRERNA1 promoter presented at E2. These results demonstrated that TFAP4 directly regulated 1ncRNA TRERNA1 transcription by targeting the E-box of its promoter region, particularly to the E2 in GC cells.

Elevated TFAP4 is positively related to metastasis in $G C$ cases. Numerous studies have revealed that TFAP4 plays a vital role in carcinogenesis and tumor development $(16,18,19)$. In this study, in order to understand the expression pattern of TFAP4 in GC tissues and the association between the expression of TFAP4 with the clinicopathological features of GC patients, we analyzed the expression of TFAP4 in 48 pairs of GC tissues and non-tumorous tissues. As revealed by RT-qPCR analysis, the expression levels of TFAP4 were markedly higher in the tumor tissues compared with the adjacent non-tumor tissues (Fig. 4), and a significant association was observed between the expression level of TFAP4, lymph node metastasis and Lauren classification in GC specimens, although no other significant associations were observed concerning the TFAP4 level and GC patients (Table I). These results indicated the potential role of TFAP4 during GC development and dissemination through the regulation of TRERNA1.

Increased expression of TRERNAI is positively related to the elevated TFAP4 level and promotes the migration and invasion ability of $G C$ cells. Since TRERNA1 was revealed to be a direct transcriptional target of TFAP4 in GC cells, we investigated whether TFAP4 influenced the expression 
$\mathbf{A}$

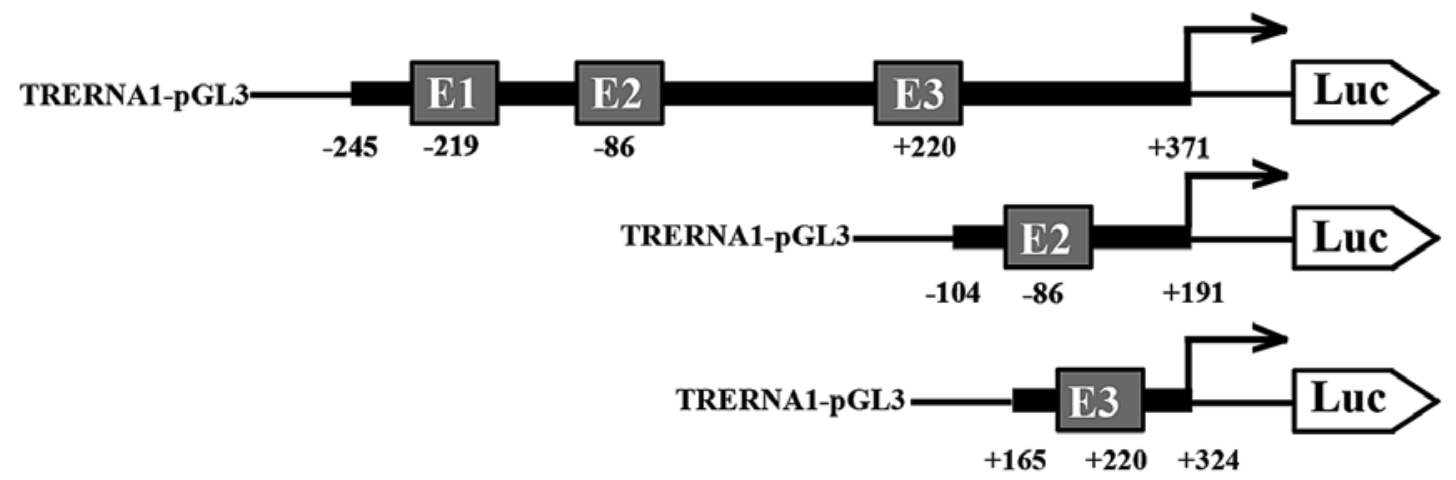

B
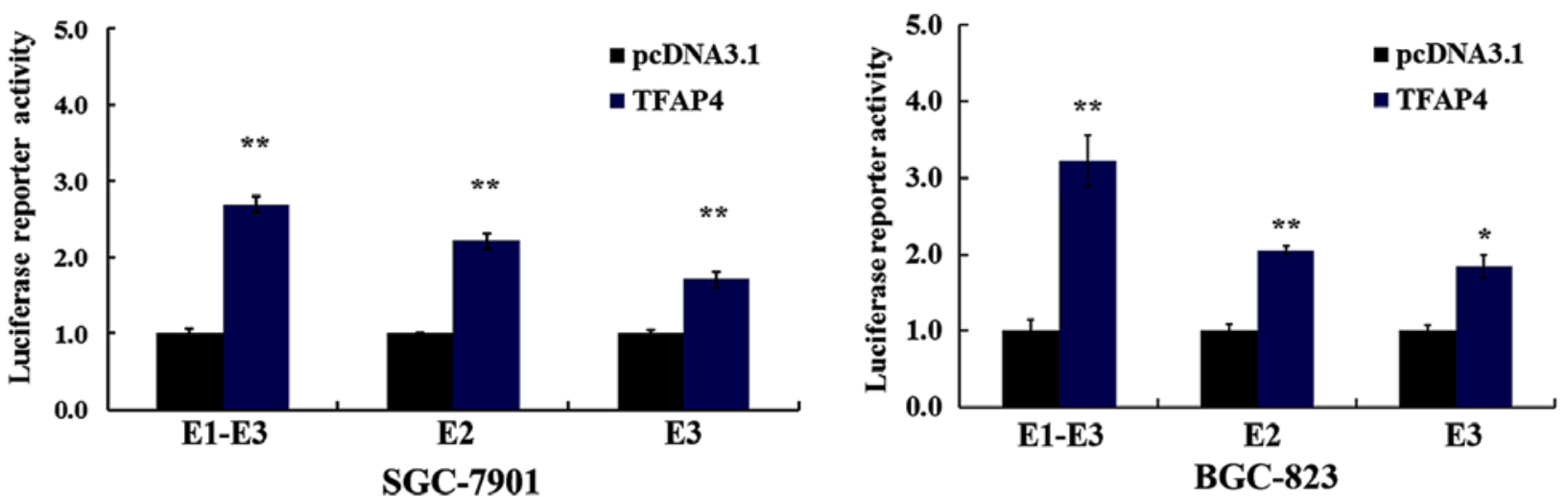

C
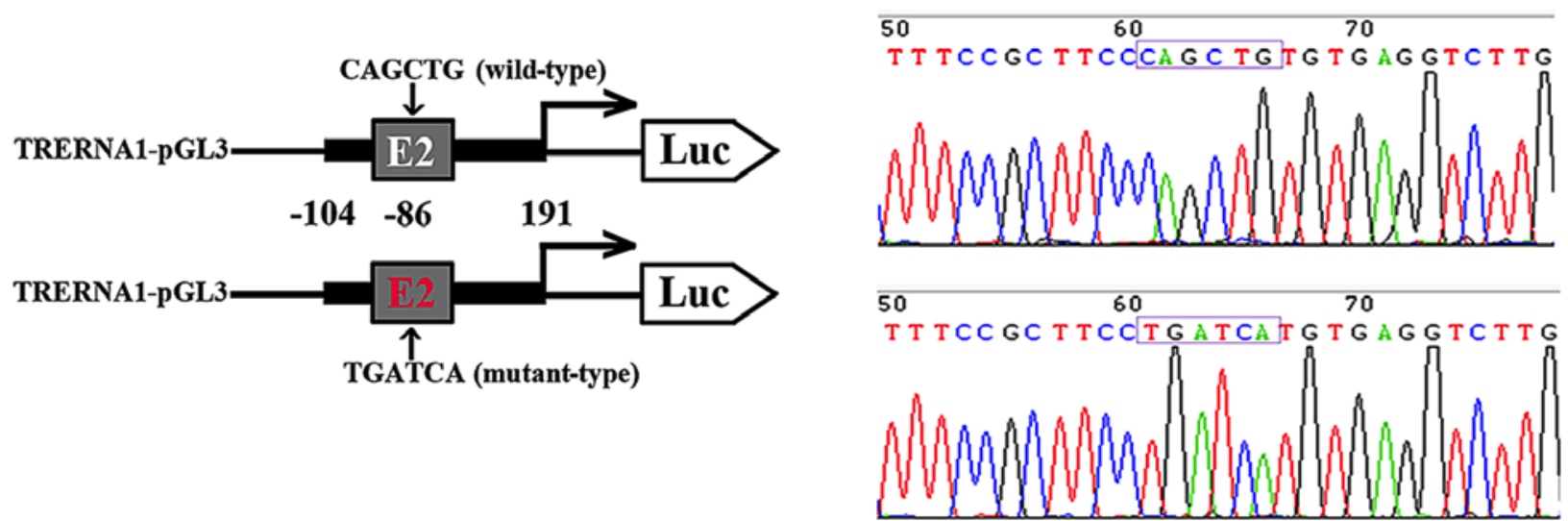

D

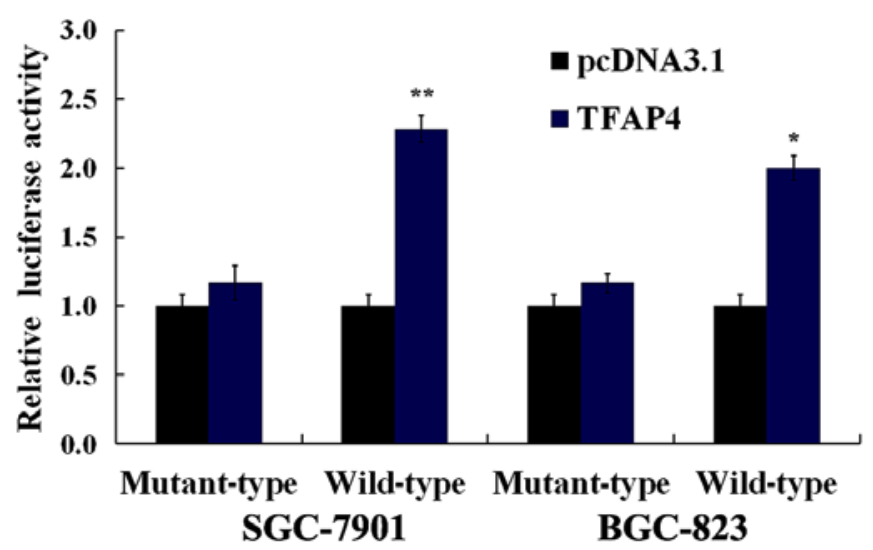

$\mathbf{E}$

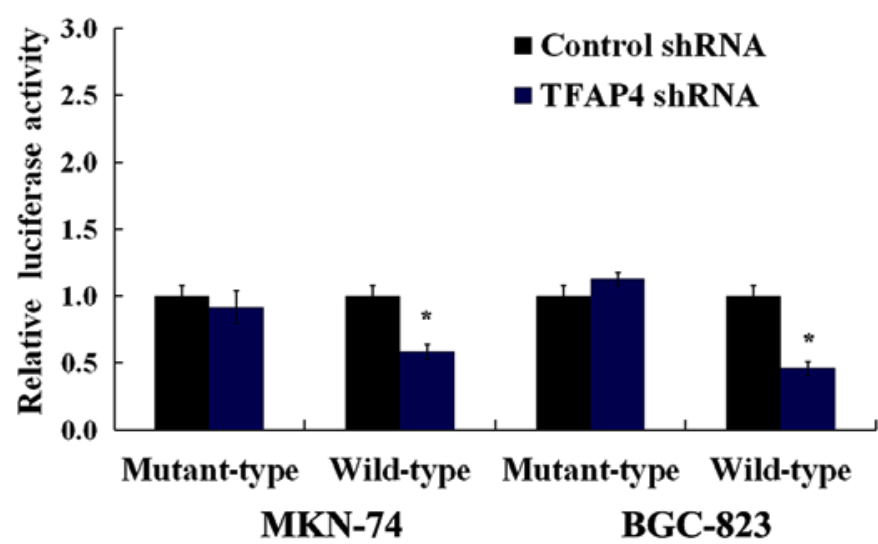

Figure 2. TFAP4 stimulates the activity of the TRERNA1 promoter in GC cells. (A) A schematic diagram depicting the construction of the dual-luciferase reporter vector with truncated fragments containing different E-boxes (E1-E3, E2 and E3) in the TRERNA1 promoter. (B) Compared with the control group, TFAP4 significantly increased the transcriptional activity of TRERNA1 promoter containing different truncated E-box fragments in SGC-7901 and BGC-823 cells by dual luciferase assays. (C) Schematic representation of the structure and the oligonucleotide sequences containing the wild-type and mutated E-box 2 motifs (E2) in the TRERNA1 promoter. (D and E) Compared with the control group, TFAP4 affected the transcriptional activity of wild-type E-box (E2), however it did not affect mutant-type E-box. Luciferase activities were determined by the dual-luciferase assay system $48 \mathrm{~h}$ after transfection in AGS, BGC-823 and MKN-74 cells. Vertical columns and error bars represent mean \pm SD of three independent experiments. ${ }^{*} \mathrm{P}<0.05,{ }^{* *} \mathrm{P}<0.01$. 

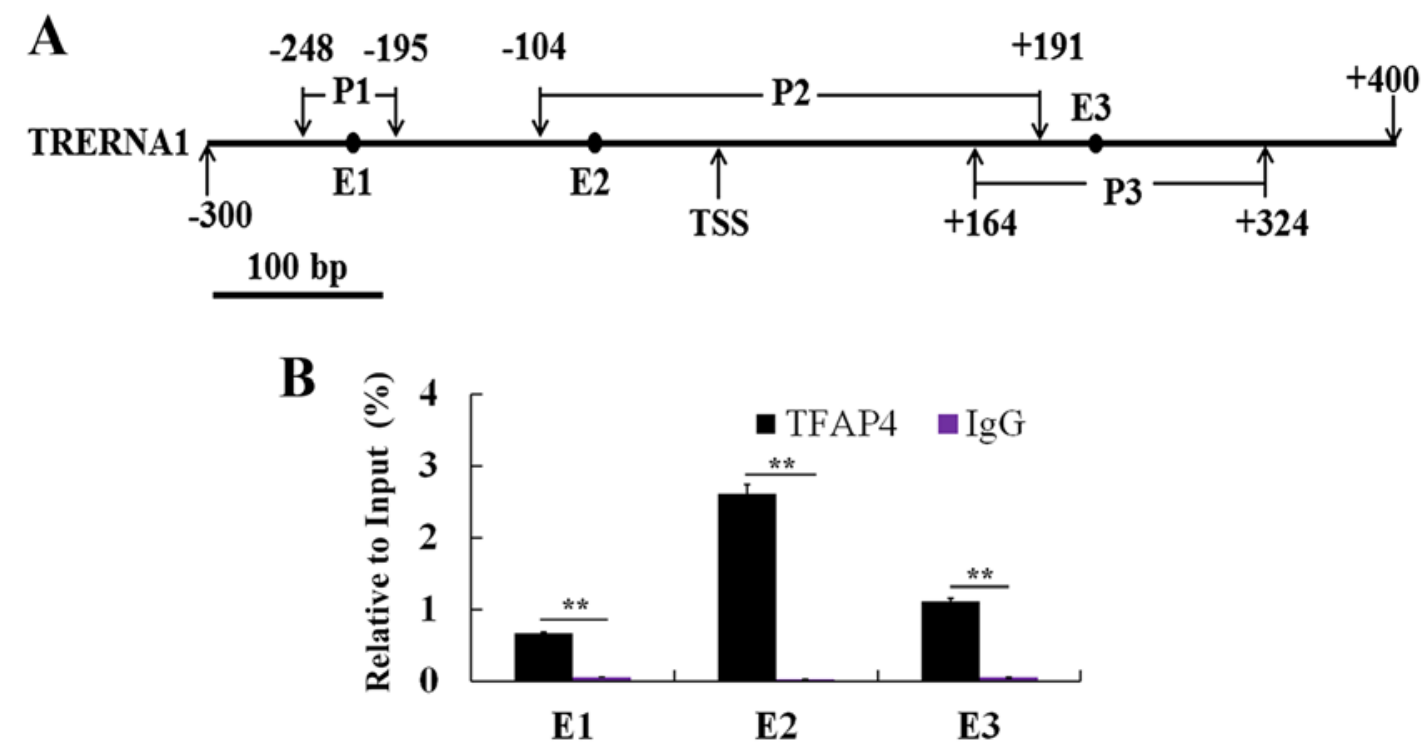

Figure 3. TFAP4 binds directly to the E-box of the TRERNA1 promoter region by ChIP assay. (A) Diagram of the promoter of the TRERNA1 gene, and the transcription start site (TSS) is indicated by an arrow. The vertical arrows indicate the location of the primer pairs (P1, P2 and P3) used for ChIP-PCR assays of E1-E3 fragments in TRERNA1 promoter. (B) The ChIP-PCR indicated that TFAP4 could bind TRERNA1 at the TFAP4-binding motif (E-box) of the TRERNA1 promoter, and the binding ability of TFAP4 to E2 was higher than that of E1 and E3. Vertical columns and error bars represent mean \pm SD. ${ }^{* *} \mathrm{P}<0.01$.

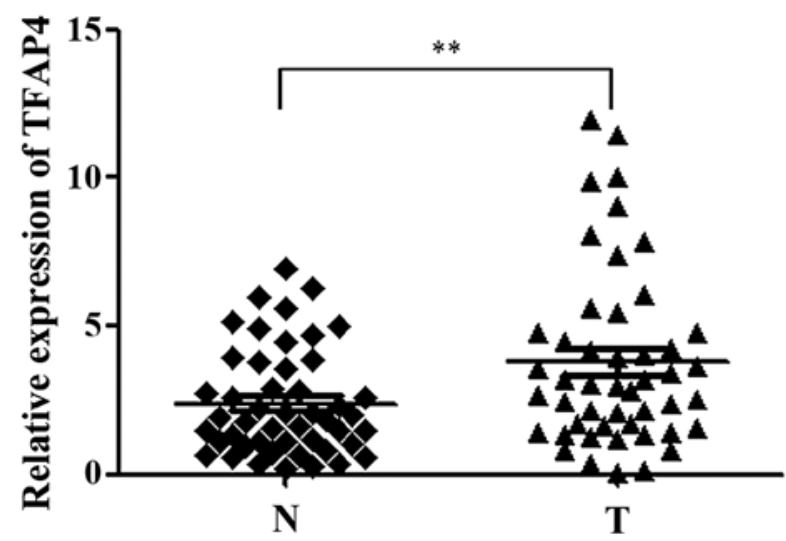

Figure 4. The expression level of TFAP4 in tumor tissues is statistically higher than that in the paired non-tumor tissues in GC cases. Bar graph represents mean $\pm \mathrm{SD}$. ${ }^{* *} \mathrm{P}<0.01$.

level of TRERNA1 in GC tissue specimens. As expected, the increased expression of TRERNA1 was observed to have a strong positive correlation with increased TFAP4 expression in GC cases (Fig. 5A). Subsequently, to assess the potential role of TRERNA1 in GC cell migration and invasion, we transfected TRERNA1 overexpression construct into BGC-823 and SGC-7901 cells, in which the expression level of TRERNA1 is relatively low among human GC cell lines (20). Wound healing assay revealed that the enforced expression of TRERNA1 in GC cells markedly enhanced the ability of the BGC-823 and SGC-7901 cells compared with the control (Fig. 5B). Similarly, the enforced expression of TRERNA1 increased the migration and invasion ability of the BGC-823 and SGC-7901 cells (Fig. 5C). These data further indicated that the increased expression level of TRERNA1 upregulated by TFAP4 promoted the migration and invasion of GC cells.

\section{Discussion}

GC is the fifth most common malignancy and the second leading cause of cancer-related mortality worldwide (21). Tumor metastasis is responsible for $\sim 90 \%$ of cancer-associated deaths, yet this process remains poorly understood (4,5,22-26). Therefore, it is urgent to uncover the pivotal genes and underlying molecular mechanisms in cancer metastasis. With the rapid development of sequencing technology, researchers found that only $2 \%$ of the human genome DNA includes protein-coding sequences (27). Non-coding RNAs, including lncRNAs have gained widespread attention as a potentially crucial molecule in disease onset and development in recent years (28), and hundreds of lncRNAs have been identified to be dysregulated in human cancers (29-31). Accumulating evidence has demonstrated that dysregulated lncRNAs, such as H19HOXA11-AS and UCA1 have already been confirmed to contribute to cell invasion and metastasis in GC (32-35).

TRERNA1 has been reported to act as a metastasis-promoting oncogene in lung and breast cancer $(36,37)$. In the present study, we observed that TRERNA1 was significantly upregulated in GC tissues compared with paired normal tissues. Data from in vitro and in vivo experiments have also indicated that TRERNA1 acts as an onco-lncRNA, which promotes the metastasis and invasion of GC cells (20). A recent study demonstrated that miR-190a inhibited epithelial-mesenchymal transition (EMT) in hepatocarcinoma cells via targeting the lncRNA TRERNA1 (38). By bioinformatics analysis, in this study, it was suggested that TFAP4 could be considered as a potential regulator of TRERNA1. In addition, a loss-of-function and gain-of-function study revealed that TFAP4 regulated the expression of TRERNA1, and double luciferase activity assay revealed that TFAP4 regulated the transcriptional activity of TRERNA1. Furthermore, ChIP-PCR revealed that TFAP4 bound directly to the E-box site of the TRERNA1 promoter to regulate its expression. 


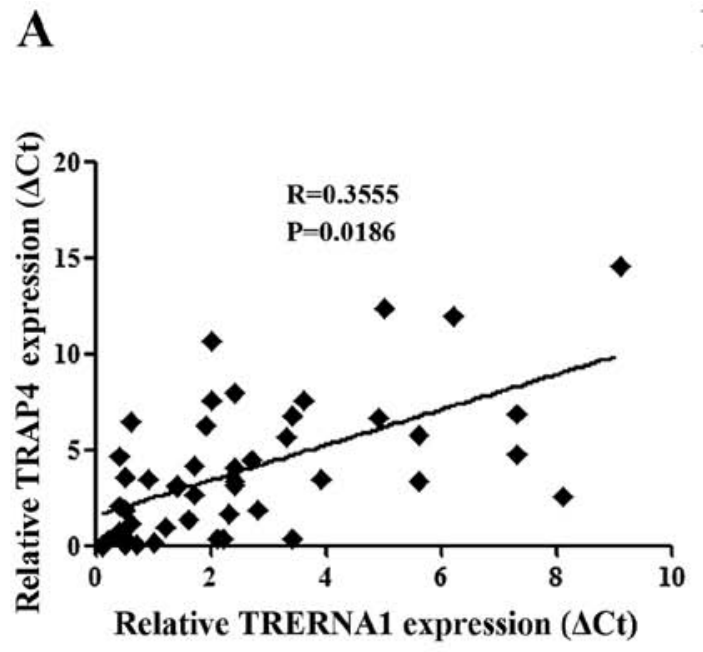

C
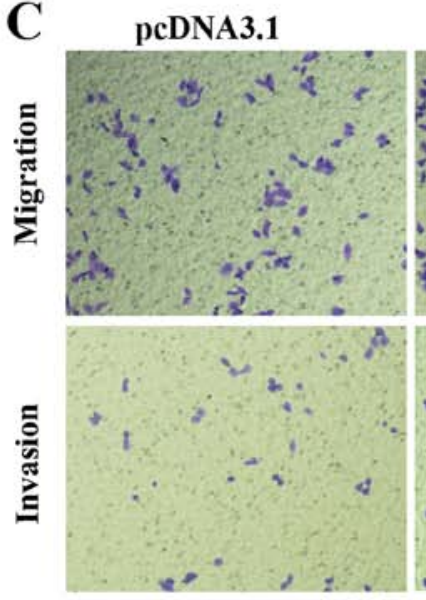

SGC-7901

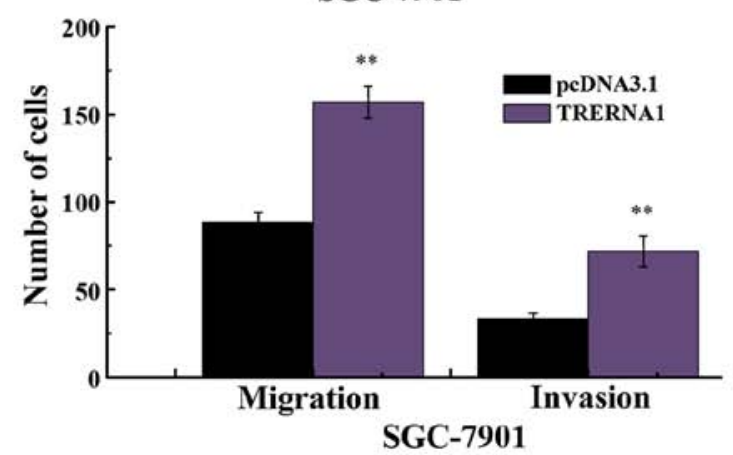

B
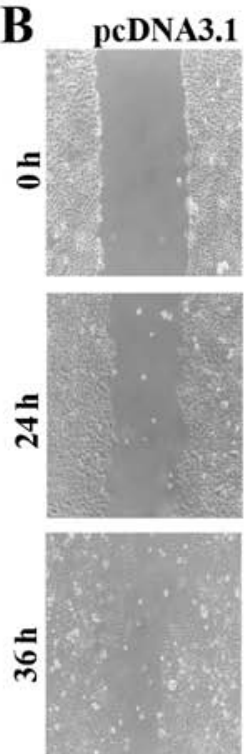

TRERNA1
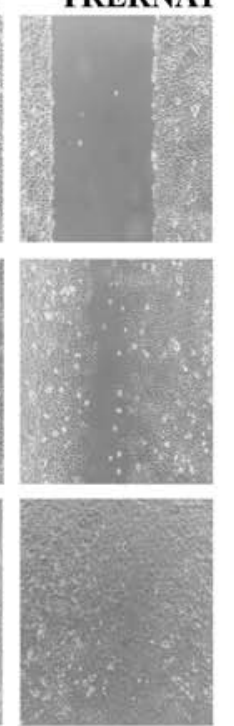

SGC-7901

pcDNA3.1
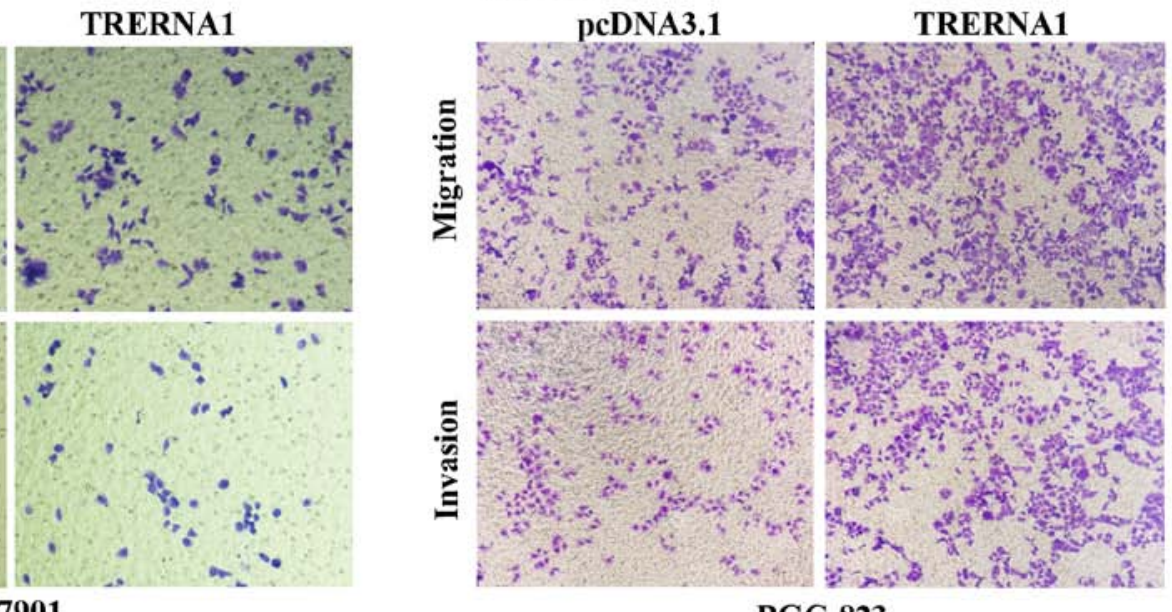

BGC-823

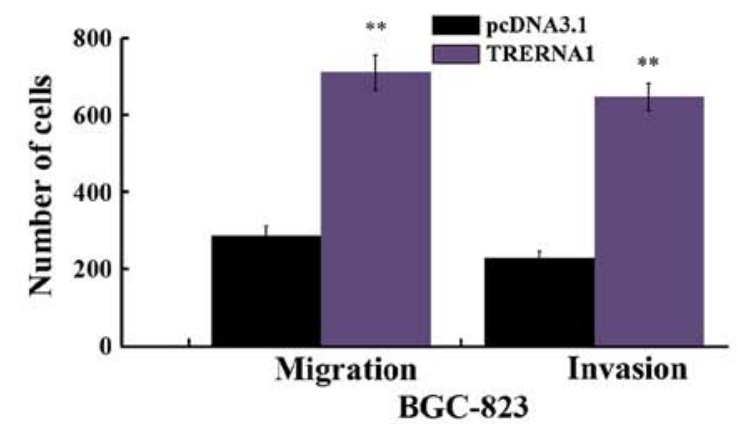

Figure 5. TRERNA1 is regulated by TFAP4 and involved in the migration and invasion of GC cells. (A) A significant positive correlation between TFAP4 and TRERNA1 gene expression in 48 clinical GC samples. (B) Compared with control group, TRERNA1 promoted the cell migration ability in SGC-7901 and BGC-823 cells by wound healing assay. (C) Compared with control group, TRERNA1 significantly promoted GC cell migration and invasion by Transwell migration and invasion assays in SGC-7901 and BGC-823 cells. Representative images of Transwell assays (left) and statistical analyses of cell invasion and migration (right). Vertical columns and error bars represent mean $\pm \mathrm{SD}$ from three independent experiments. ${ }^{* *} \mathrm{P}<0.01$.

We also observed that the expression level of TFAP4 was significantly related to the expression level of TRERNA1 in GC cases. These results indicated that TFAP4 regulated the expression of TRERNA1 in gastric carcinogenesis.

TFAP4 belongs to the helix-loop-helix family and plays an important regulatory role by binding to the conserved E-box (CAGCTG) sequence in transcriptional networks, thus affecting cell proliferation, differentiation, cell lineage determination and other essential biological processes (39). Previous studies have revealed that TFAP4 regulates the expression of genes such as p21 and p16 (17,40), caspase-9 (41) and HDM2 (42). According to recent research, the overexpression of TFAP4 has been reported in colorectal, breast, lung and prostate cancer. TFAP4 has been shown to induce EMT 
and enhance the migration and invasion of colorectal cancer cells $(16-18,40,43)$. In GC, research has revealed that the expression of TFAP4 is significantly higher in tumor tissue than in non-neoplastic tissue, and an elevated TFAP4 expression significantly and positively correlates with the degree of tumor differentiation, depth of tumor invasion, lymph node metastasis and TNM stage (19). The downregulation of TFAP4 has been shown to inhibit proliferation, induce cell-cycle arrest and promote the apoptosis of GC cells (44). TFAP4 performs critical functions in GC by participating in signaling pathways such as the ECM-receptor-interaction and spliceosome (45). Therefore, the function and molecular mechanisms of TFAP4 in gastric carcinogenesis warrant further investigation.

The genome-wide characterization of TFAP4 DNA binding sites and genes directly regulated by TFAP4 were identified using ChIP, next-generation sequencing and bioinformatics analysis (16). These studies on TFAP4-mediated transcriptional regulation have mainly focused on protein-coding genes; however the regulation of TFAP4 to lncRNAs has not yet been investigated. In the present study, our findings indicated that upregulated lncRNA TRERNA1 expression, which could contribute to gastric cell invasion and metastasis was induced by TFAP4 by directly binding to the CACGTG motifs in the promoter of TRERNA1. The present study clarified the mechanism of upregulated TRERNA1, which was involved in the invasion and metastasis of GC, and also revealed a new field of TFAP4 regulation of the expression of IncRNA in tumorigenesis. Furthermore, our study indicated that the TFAP4-TRERNA1 axis may be a promising novel therapeutic target for the intervention therapy of GC.

\section{Acknowledgements}

Not applicable.

\section{Funding}

The present study was supported by the National Natural Science Foundation of China (nos. 81672414 and 81472548), the Jiangsu Provincial Natural Science Foundation-Youth Foundation (BK20160667), the Foundational Research Funds for the Central University (2242016k41034) and the Foundation for Young Talents and Natural Science in Higher Education of Anhui Province (KJ2017A213, gxyq2018035).

\section{Availability of data and materials}

The datasets used and/or analyzed during the current study are available from the corresponding author on reasonable request.

\section{Authors' contributions}

HF conceived and designed the study. HW performed the experiments and wrote the manuscript. XL, PG and WS provided assistance for clinical sample collection, preservation, data analysis and statistical analysis. MZ, YL and ZZ were involved in interpreting the results, manuscript editing and manuscript review. All authors have read and approved the content of the manuscript.

\section{Ethics approval and consent to participate}

The study was approved by the Research Ethics Committee of Southeast University, and written informed consent was obtained from all patients.

\section{Consent for publication}

Not applicable.

\section{Competing interests}

The authors declare that they have no competing interests.

\section{References}

1. Chen W, Zheng R, Baade PD, Zhang S, Zeng H, Bray F, Jemal A, Yu XQ and He J: Cancer statistics in China, 2015. CA Cancer J Clin 66: 115-132, 2016.

2. Cancer Genome Atlas Research Network: Comprehensive molecular characterization of gastric adenocarcinoma. Nature 513: 202-209, 2014.

3. Ferlay J, Soerjomataram I, Dikshit R, Eser S, Mathers C, Rebelo M, Parkin DM, Forman D and Bray F: Cancer incidence and mortality worldwide: Sources, methods and major patterns in GLOBOCAN 2012. Int J Cancer 136: E359-E386, 2015.

4. Chaffer CL and Weinberg RA: A perspective on cancer cell metastasis. Science 331: 1559-1564, 2011.

5. Kapranov P, Cheng J, Dike S, Nix DA, Duttagupta R, Willingham AT, Stadler PF, Hertel J, Hackermuller J, Hofacker IL, et al: RNA maps reveal new RNA classes and a possible function for pervasive transcription. Science 316: 1484-1488, 2007.

6. Wang KC and Chang HY: Molecular mechanisms of long noncoding RNAs. Mol Cell 43: 904-914, 2011.

7. Guttman M, Donaghey J, Carey BW, Garber M, Grenier JK, Munson G, Young G, Lucas AB, Ach R, Bruhn L, et al: lincRNAs act in the circuitry controlling pluripotency and differentiation. Nature 477: 295-300, 2011.

8. Rinn JL, Kertesz M, Wang JK, Squazzo SL, Xu X, Brugmann SA, Goodnough LH, Helms JA, Farnham PJ, Segal E and Chang HY: Functional demarcation of active and silent chromatin domains in human HOX loci by noncoding RNAs. Cell 129: 1311-1323, 2007.

9. Cesana M, Cacchiarelli D, Legnini I, Santini T, Sthandier O, Chinappi M, Tramontano A and Bozzoni I: A long noncoding RNA controls muscle differentiation by functioning as a competing endogenous RNA. Cell 147: 358-369, 2011.

10. Xing Z, Lin A, Li C, Liang K, Wang S, Liu Y, Park PK, Qin L, Wei Y, Hawke DH, et al: IncRNA directs cooperative epigenetic regulation downstream of chemokine signals. Cell 159: 1110-1125, 2014.

11. Heo JB and Sung S: Vernalization-mediated epigenetic silencing by a long intronic noncoding RNA. Science 331: 76-79, 2011.

12. Jariwala $N$ and Sarkar D: Emerging role of lncRNA in cancer: A potential avenue in molecular medicine. Ann Transl Med 4: 286, 2016.

13. Livak KJ and Schmittgen TD: Analysis of relative gene expression data using real-time quantitative PCR and the $2^{-\Delta \Delta C_{\mathrm{T}}}$ method. Methods 25: 402-408, 2001.

14. Cui H, Wang L, Gong P, Zhao C, Zhang S, Zhang K, Zhou R, Zhao Z and Fan H: Deregulation between miR-29b/c and DNMT3A is associated with epigenetic silencing of the CDH1 gene, affecting cell migration and invasion in gastric cancer. PLoS One 10: e0123926, 2015.

15. Karin M: Too many transcription factors: Positive and negative interactions. New Biol 2: 126-131, 1990.

16. Jackstadt R, Röh S, Neumann J, Jung P, Hoffmann R, Horst D, Berens C, Bornkamm GW, Kirchner T, Menssen A, et al: AP4 is a mediator of epithelial-mesenchymal transition and metastasis in colorectal cancer. J Exp Med 210: 1331-1350, 2013.

17. Jackstadt R, Jung P and Hermeking H: AP4 directly downregulates p16 and p21 to suppress senescence and mediate transformation. Cell Death Dis 4: e775, 2013. 
18. Gong $\mathrm{H}$, Han S, Yao H, Zhao $\mathrm{H}$ and Wang Y: AP4 predicts poor prognosis in nonsmall cell lung cancer. Mol Med Rep 10: 336-340, 2014.

19. Xinghua L, Bo Z, Yan G, Lei W, Changyao W, Qi L, Lin Y, Kaixiong T, Guobin W and Jianying C: The overexpression of AP-4 as a prognostic indicator for gastric carcinoma. Med Oncol 29: 871-877, 2012.

20. Wu H, Hu Y, Liu X, Song W, Gong P, Zhang K, Chen Z, Zhou M, Shen X, Qian Y and Fan H: LncRNA TRERNAl function as an enhancer of SNAII promotes gastric cancer metastasis by regulating epithelial-mesenchymal transition. Mol Ther Nucleic Acids 8: 291-299, 2017.

21. Torre LA, Bray F, Siegel RL, Ferlay J, Lortet-Tieulent J and Jemal A: Global cancer statistics, 2012. CA Cancer J Clin 65 87-108, 2015.

22. Gupta GP and Massagué J: Cancer metastasis: Building a framework. Cell 127: 679-695, 2006

23. Hanahan D and Weinberg RA: Hallmarks of cancer: The next generation. Cell 144: 646-674, 2011.

24. Hanahan D and Weinberg RA: The hallmarks of cancer. Cell 100: 57-70, 2000.

25. Yang SY, Miah A, Pabari A and Winslet M: Growth factors and their receptors in cancer metastases. Front Biosci 16: 531-538, 2011.

26. Jemal A, Bray F, Center MM, Ferlay J, Ward E and Forman D: Global cancer statistics. CA Cancer J Clin 61: 69-90, 2011.

27. Kung JT, Colognori D and Lee JT: Long noncoding RNAs: Past, present, and future. Genetics 193: 651-669, 2013.

28. Heery R, Finn SP, Cuffe S and Gray SG: Long non-coding RNAs: Key regulators of epithelial-mesenchymal transition, tumour drug resistance and cancer stem cells. Cancers 9: E38, 2017.

29. Prensner JR and Chinnaiyan AM: The emergence of lncRNAs in cancer biology. Cancer Discov 1: 391-407, 2011.

30. Ning S, Zhang J, Wang P, Zhi H, Wang J, Liu Y, Gao Y, Guo M, Yue M, Wang L and Li X: Lnc2Cancer: A manually curated database of experimentally supported lncRNAs associated with various human cancers. Nucleic Acids Res 44: D980-D985, 2016

31. Gibb EA, Brown CJ and Lam WL: The functional role of long non-coding RNA in human carcinomas. Mol Cancer 10: 38, 2011.

32. Wang ZQ, He CY, Hu L, Shi HP, Li JF, Gu QL, Su LP, Liu BY, $\mathrm{Li} \mathrm{C}$ and $\mathrm{Zhu} \mathrm{Z}$ : Long noncoding RNA UCA1 promotes tumour metastasis by inducing GRK2 degradation in gastric cancer. Cancer Lett 408: 10-21, 2017.

33. Gutschner T and Diederichs S: The hallmarks of cancer: A long non-coding RNA point of view. RNA Biol 9: 703-719, 2012.
34. Sun M, Nie F, Wang Y, Zhang Z, Hou J, He D, Xie M, Xu L, De W, Wang $Z$ and Wang J: LncRNA HOXA11-as promotes proliferation and invasion of gastric cancer by scaffolding the chromatin modification factors PRC2, LSD1, and DNMT1. Cancer Res 76: 6299-6310, 2016.

35. Li H, Yu B, Li J, Su L, Yan M, Zhu Z and Liu B: Overexpression of lncRNA H19 enhances carcinogenesis and metastasis of gastric cancer. Oncotarget 5: 2318-2329, 2014.

36. Ørom UA, Derrien T, Beringer M, Gumireddy K, Gardini A, Bussotti G, Lai F, Zytnicki M, Notredame C, Huang Q, et al: Long noncoding RNAs with enhancer-like function in human cells. Cell 143: 46-58, 2010.

37. Gumireddy K, Li AP, Yan JC, Setoyama T, Johannes GJ, Orom UA, Tchou J, Liu Q, Zhang L, Speicher DW, et al: Identification of a long non-coding RNA-associated RNP complex regulating metastasis at the translational step. EMBO J 32: 2672-2684, 2013.

38. Wang X, Ren Y, Yang X, Xiong X, Han S, Ge Y, Pan W, Zhou L, Yuan Q and Yang M: miR-190a inhibits epithelial-mesenchymal transition of hepatoma cells via targeting the long non-coding RNA treRNA. FEBS Lett 589: 4079-4087, 2015.

39. Atchley WR and Fitch WM: A natural classification of the basic helix-loop-helix class of transcription factors. Proc Natl Acad Sci USA 94: 5172-5176, 1997.

40. Jung P, Menssen A, Mayr D and Hermeking H: AP4 encodes a c-MYC-inducible repressor of $p 21$. Proc Natl Acad Sci USA 105: 15046-15051, 2008.

41. Tsujimoto K, Ono T, Sato M, Nishida T, Oguma T and Tadakuma T: Regulation of the expression of caspase- 9 by the transcription factor activator protein-4 in glucocorticoid-induced apoptosis. J Biol Chem 280: 27638-27644, 2005.

42. Ku WC, Chiu SK, Chen YJ, Huang HH, Wu WG and Chen YJ: Complementary quantitative proteomics reveals that transcription factor AP-4 mediates E-box-dependent complex formation for transcriptional repression of $H D M 2$. Mol Cell Proteomics 8: 2034-2050, 2009.

43. Cao J, Tang M, Li WL, Xie J, Du H, Tang WB, Wang H, Chen XW, Xiao $\mathrm{H}$ and Li Y: Upregulation of activator protein-4 in human colorectal cancer with metastasis. Int J Surg Pathol 17: 16-21, 2009.

44. Liu X, Zhang B, Guo Y, Liang Q, Wu C, Wu L, Tao K, Wang G and Chen J: Down-regulation of AP-4 inhibits proliferation, induces cell cycle arrest and promotes apoptosis in human gastric cancer cells. PLoS One 7: e37096, 2012.

45. Wang Y: Transcriptional regulatory network analysis for gastric cancer based on mRNA microarray. Pathol Oncol Res 23. 785-791, 2017. 